Two iconicity measures are better than one

\title{
Two measures are better than one: Combining iconicity ratings and guessing experiments for a more nuanced picture of iconicity in the lexicon
}

\author{
Bonnie McLean ${ }^{*}$, Michael Dunn' ${ }^{1}$, Mark Dingemanse ${ }^{2}$ \\ 'Uppsala University, Sweden, Department of Linguistics and Philology \\ ${ }^{2}$ Radboud University, The Netherlands, Centre for Language Studies \\ *Corresponding author: bonnie.mclean@lingfil.uu.se \\ Abstract
}

11 Iconicity in language is receiving increased attention from many fields, but our understanding of

12 iconicity is only as good as the measures we use to quantify it. We collected iconicity measures

13 for 304 Japanese words from English-speaking participants, using rating and guessing tasks. The

14 words included ideophones (structurally marked depictive words) along with regular lexical

15 items from similar semantic domains (e.g. fuwafuwa 'fluffy', jawarakai 'soft'). The two

16 measures correlated, speaking to their validity. However, ideophones received consistently

17 higher iconicity ratings than other items, even when guessed at the same accuracies, suggesting

18 the rating task is more sensitive to cues like structural markedness that frame words as iconic.

19 These cues did not always guide participants to the meanings of ideophones in the guessing task,

20 but they did make them more confident in their guesses, even when they were wrong.

21 Consistently poor guessing results reflect the role different experiences play in shaping

22 construals of iconicity. Using multiple measures in tandem allows us to explore the interplay

23 between iconicity and these external factors. To facilitate this, we introduce a reproducible

24 workflow for creating rating and guessing tasks from standardised wordlists, while also making

25 improvements to the robustness, sensitivity and discriminability of previous approaches.

26 Keywords: iconicity, ideophones, lexical norms, iconicity ratings, guessability 
Two iconicity measures are better than one

\section{Introduction}

28 What does it mean when we say that something is a "good word for" a concept? Certain words,

29 like klutz, smooch, and smoosh, seem to fit their meanings. We may have a sense that the French

30 belle is a more appropriate word for BEAUTY than the Swedish vacker. Such intuitions are

31 likely to be somewhat subjective, and influenced by our own experiences, but we all have them.

32 This quality of resemblance between forms and meanings is known as iconicity.

33 Iconicity-defined here as a perceived resemblance between aspects of form and

34 meaning-is now increasingly recognised as a key property of language (see e.g. Dingemanse et

35 al., 2015; Perniss et al., 2010; Perniss \& Vigliocco, 2014 for reviews). But where do perceptions

36 of iconicity come from? From the very earliest investigations of iconicity in language, there has

37 been an assumption that perceptions of iconicity arise from formal correspondences. The

38 presence of /i/ leads to perception of smallness, /a/ to largeness (Sapir, 1929); sonorants are

39 curved, obstruents are spiked (Köhler, 1929), and so on and so forth. The decoding of this 'iconic

40 language' has been an appealing challenge, and the continuation of this line of work has proved

41 fruitful; many more such iconic correspondences have now been uncovered.

42 Iconicity can be seen at the level of the linguistic system as a whole, by identifying

43 skewed distributions of form-meaning mappings in lexicons, both within a single language (e.g.

44 Monaghan et al., 2014; Winter \& Perlman, 2021a), and across languages (Blasi et al., 2016;

45 Johansson et al., 2020; Joo, 2020; e.g. Wichmann et al., 2010; Winter et al., 2022). Iconicity can

46 also be seen on an individual level, in behavioural paradigms like iconicity rating tasks (Caselli

47 et al., 2017; Perry et al., 2015; Thompson et al., 2020; e.g. Vinson et al., 2008) and guessing

48 experiments (Brown et al., 1955; Dingemanse et al., 2016; Köhler, 1929; Ramachandran \&

49 Hubbard, 2001; e.g. Sapir, 1929; Tsuru \& Fries, 1933). One advantage of studying individual 
Two iconicity measures are better than one

50 construals of iconicity in behavioural tasks is that we can manipulate elements of these tasks to

51 explore factors that shape construals of iconicity. Several studies have now found discrepancies

52 emerge between different populations and tasks when exploring construals of iconicity, which

53 suggest that this is more than just a simple 'decoding' process. At the same time, it is becoming

54 increasingly apparent that the associations language users tap into in interpreting iconicity do not

55 stem from a single, universal iconic 'language', but rather from many iconic 'languages' that

56 vary between individuals and evolve with experience.

57 As an illustration, Occhino et al. (2017) show that perceptions of iconicity are influenced

58 by familiarity, with sign language users consistently rating signs from their own language as

59 more iconic than signs from a foreign language. Construals of iconicity also have a

60 developmental trajectory, as older children are better at recognising iconicity than younger

61 children (Fernández-Prieto et al., 2015; Fort et al., 2018; Magid \& Pyers, 2017; e.g. Namy, 2008;

62 Tzeng et al., 2017). Adult speakers of different languages exhibit differing sensitivities to

63 specific form-meaning associations (Fordyce, 1988; Iwasaki et al., 2007; Kwon, 2017; Shinohara

64 \& Kawahara, 2010; Taylor \& Taylor, 1962). For example, Iwasaki and colleagues (2007) asked

65 both Japanese and English speaking participants to rate Japanese ideophones for manners of

66 walking along several semantic differential scales. They found that while Japanese speakers

67 associated ideophones containing voiceless consonants with a lighter gait, and ideophones

68 containing voiced consonants with a heavier gait, English speakers were only sensitive to the

69 latter association. The authors conclude that the iconicity of at least some Japanese ideophones

70 exhibits a degree of language specificity, meaning that it is something Japanese speakers learn

71 through exposure to distributional patterns of sound-meaning pairings in their own lexicon, 
Two iconicity measures are better than one

72 rather than a property that is immediately apparent from the sound of the word alone. Akita and

73 Imai (2022) term this emergent iconicity.

Other studies vary aspects of task design to explore the role that external factors like

75 context and framing play in shaping construals of iconicity. For example, two studies found that

76 participants were better at guessing the meanings of foreign words when pronounced with an

77 expressive intonation, compared to a flat intonation (Dingemanse et al., 2016; Kunihira, 1971).

78 Similar work by Sevcikova Sehyr \& Emmorey found discrepancies in construals of iconicity

79 between different tasks. They observed that while participants may rate a sign as highly iconic in

80 a task where the meaning is provided, the same sign could be considered only weakly transparent

81 in a guessing task (Sevcikova Sehyr \& Emmorey, 2019). Clearly, further work is needed to

82 understand what it is that we really measure when we measure iconicity, and to learn how to

83 untangle the contributions of form-meaning resemblances from other factors that influence

84 perceptions of form-meaning fit (for more on this see e.g. Motamedi et al., 2019; Winter \&

85 Perlman, 2021b).

The current study embraces this challenge. We collected iconicity measures for 304

87 Japanese words from monolingual English speaking participants, in both an iconicity rating task

88 and a series of guessing experiments, to better understand the role that factors external to form-

89 meaning resemblances per se (e.g. native language, task design) play in shaping construals of

90 iconicity. It is important to understand these factors, so that they can be taken into account when

91 collecting measures of iconicity to use as explanatory variables in other analyses. In collecting

92 these different measures, we made some variations particularly to the design of the guessing

93 experiments in order to fine-tune the sensitivity of this task to detecting form-meaning

94 resemblances specifically. While individual measures can be fine-tuned in this way to focus on 
Two iconicity measures are better than one

95 specific factors, our experience of iconicity in the real world is multidimensional (e.g. Hodge \&

96 Ferrara, 2022), and for this reason we believe that moving forward triangulating insights from

97 multiple measures offers the greatest explanatory power when seeking to understand how

98 iconicity affects various aspects of language. We illustrate this with the guessing and rating data

99 by showing how when used together the two measures shed light on each other, and on the

100 contribution of different factors to iconic effects.

101 As a final contribution, to make it easier for future studies to use these measures in

102 tandem, we introduce a reproducible workflow for generating rating and guessing tasks from a

103 single wordlist, with support for a variety of stimulus formats (audio, video, and images). This is

104 available in an open source python package, icotools (https://pypi.org/project/icotools/), to

105 make the collection of behavioural measures of iconicity quicker, easier, and more comparable

106 between future studies.

\section{Method}

108 The materials used to produce the experiments, as well as the resulting data and analyses are

109 available on the Open Science Framework, at https://osf.io/j57uc/.

\section{$110 \quad 2.1 \quad$ Materials}

111 The materials were 304 Japanese words taken from the Japonic Sensory Lexicon database

112 (McLean 2022), which is a collection of words for 110 sensory concepts in 49 Japonic varieties.

113 The words in the database were collated from three different sources: Sanada and Tomosada

114 (2015, 2018), and Hirayama (1992). Any available concepts related to the senses were included.

115 For all three sources, words for each concept were collected from speakers of different Japonic

116 varieties by asking the speakers to translate sentences from the standard variety into their local 
Two iconicity measures are better than one

117 variety. The iconicity measures in this study were collected to enrich the data in the Japonic

118 Sensory Lexicon, as a prior step in a broader investigation of this lexicon. Further information

119 about the Japonic Sensory Lexicon can be found in the Supplementary Materials.

120 The words come from the native stratum of the Japanese lexicon, which can be further

121 divided into two substrata: the prosaic and the ideophonic (also called the mimetic) stratum (see

122 e.g. Kageyama \& Kishimoto, 2016). The ideophonic stratum is defined on the basis of its

123 distinctive morphophonological properties, as well as its use of sound symbolism (Akita, 2008,

124 2017). Words belonging to the ideophonic stratum are generally considered to be iconic (Akita,

125 2008; Thompson et al., 2020), and typically depict sensory experiences (Akita, 2017). The term

126 'prosaic' is used in this study to distinguish other native words belonging to the sensory lexicon

127 of Japonic from ideophones. Frequently, a similar concept can be expressed either by a prosaic

128 word or by an ideophone. For example, something that is soft could be described in Standard

129 Japanese as jawarakai, to use a prosaic word, or fuwafuwa, to use an ideophone. This lexical

130 stratification predates the diversification of the Japonic languages from Proto-Japonic, and is

131 found in all the modern Japonic varieties used in this study. While ideophones are felt to be

132 iconic to Japanese speakers, we were interested to test if this would also be true for our English

133 speaking participants.

134 In all the experiments, the Japanese words were presented to participants as audio files.

135 This was to guard against any interference from orthography. For example, to avoid participants

136 interpreting the phonetic values of the transliterations of Japanese characters incorrectly. The

137 audio files were synthesised using the Google Cloud Text-to-Speech API

138 (https://cloud.google.com/text-to-speech), with the female Japanese Wavenet voice ja-JP-

139 Wavenet-B. They were then edited using Praat (Boersma \& Weenink, 2020) to have a flat pitch 
Two iconicity measures are better than one

140 of $200 \mathrm{~Hz}$. Since expressive prosody can enhance performance in guessing tasks (Dingemanse et

141 al., 2016; Kunihira, 1971), standardising the intonation in the recordings ensured comparisons

142 between the words were fair and not influenced by the prosody in a particular recording. We also

143 considered editing the words to have a marked intonation, but it was difficult to do this in a

144 comparable way for words of different lengths. While flat, synthetic voices may be the best

145 option for comparability, a consequence of this methodological decision is that our results almost

146 certainly underestimate the iconicity of these words in natural settings, which involve not only

147 speech segments but also prosody and accompanying multimodal cues (e.g. gesture, facial

148 expressions).

\subsection{Participants}

150689 monolingual English speakers were recruited through the crowd sourcing service Prolific

151 (www.prolific.co), and received $£ 0.75$ for their participation (based on Prolific's 'good' hourly

152 rate of $£ 7.50)$. All participants confirmed that they had no prior knowledge of Japanese.

153 Although iconicity rating tasks are usually conducted with native speakers, we used English

154 speakers for the rating task as well as the guessing experiments so that we could assess the

155 agreement between these two different measures of iconicity when collected from the same

156 population. Using non-speakers was also important as our data contained a mix of Standard

157 Japanese and non-standard Japanese words (see Section 2.1), and we were concerned that if our

158 participants were Standard Japanese speakers this would bias the Standard Japanese words

159 (Occhino et al., 2017). 
Two iconicity measures are better than one

160

161

162

163

164

165

166

167

168

169

170

171

172

173

174

175

176

177 performance on the control items, were used to identify and exclude results from participants

178 who were likely not performing the task as intended. For the rating study, we also calculated the

179 person-total correlation for each participant as an additional quality check, to identify

180 participants whose response patterns differed from those of other participants with the same

181 wordlist (following Curran, 2016). Based on these criteria, data from 11 out of 689 participants 
Two iconicity measures are better than one

182 was excluded across the different studies. Further information on these exclusions is provided in 183 the Supplementary Materials.

184 Excluding the practice and control items (which every participant saw), the final dataset 185 for the pilot guessing experiment included a median of 40 guesses per word (range: 15-129); in 186 the second guessing experiment there was a median of 30 guesses per word (range: 29-60); and 187 in the rating task there was a median of 15 ratings per word (range: 14-58). Motamedi et al. 188 (2019) suggest a benchmark of 10 ratings per word is sufficient. Since the words were presented as audio files, participants were instructed to use

190 headphones in every study, and could not proceed to the main task without first completing a 191 listening test designed to require the use of headphones (Woods et al., 2017). The response

192 buttons for each trial did not appear until after the audio files had finished playing, ensuring that 193 participants could not respond without first listening to the audio files. For every trial, the time 194 (in milliseconds) participants took to select a response for the trial (after the response buttons 195 appeared on the screen) was recorded.

196 The remainder of this section outlines the design of the three studies themselves, which 197 included: (1) a pilot study of guessing experiments following previous work (Dingemanse et al., 198 2016), (2) a series of improved guessing experiments addressing complications encountered with 199 (1), and (3) a rating task. We compare the measures of iconicity collected in these studies for 200 their robustness to different experimental choices, their sensitivity to iconicity, and their ability 201 to distinguish between different levels of iconicity in the Results section of the manuscript. 
Two iconicity measures are better than one

202

203 Following previous studies (e.g. Dingemanse et al., 2016), we piloted guessing experiments in

204 which participants were presented with a Japanese word and asked to guess its meaning from a

205 choice of two possible translations. Some of the ideophones in the data had very specific

206 meanings, which were difficult to capture in a single English word. To provide more context, the

207 translations were presented in sentence frames, with the translation word in capitals. The

208 instructions were as follows:

217 A sample trial is shown in Figure 2.1.
We are interested in how well people can guess the meanings of words in foreign languages. In this experiment, we are interested in your intuitions about the meanings of JAPANESE words. You will hear 25 Japanese words. After listening to each word, try to guess its meaning from a choice of two possible English translations. For context, meanings are given in a sentence, with the intended meaning in CAPITALS. For example, if you think the word could mean BRIGHT in the sentence 'The sun is BRIGHT', choose that sentence. If you think it could mean ROUND in the sentence, 'The sun is ROUND', choose that sentence. Trust your gut, and good luck! 
Two iconicity measures are better than one

Listen to the Japanese word, and guess its meaning. For context, meanings are given in a sentence, with the meaning in CAPITALS.

$\rightarrow 0: 00 / 0: 00 \longrightarrow$ -

After going for a run on a full stomach, my stomach was CRAMPING.

After being in the smoky room for hours, my throat felt SCRATCHY.

Figure 2.1: Guessing task, word to meaning design.

The same formulaic structure, "After X, Y”, was used for all the sentences-where $\mathrm{Y}$ is

221 the target perception, and $\mathrm{X}$ is the event that causes it. As some sensory modalities (e.g. sound)

222 may better lend themselves to the construal of iconicity than others (e.g. taste) (Perlman et al.

223 2018; Little et al. 2018), correct translations were always paired with foil translations from the

224 same modality (e.g. sound translations were only paired with other sound translations, and taste

225 translations with other taste translations, etc.). This ensured that one translation was not more

226 likely to be chosen over another simply because it belonged to a domain typically high in

227 iconicity. Only words that were neither synonyms nor antonyms of the correct translation were

228 used as foil translations. For example, in the sample trial shown in Figure 2.1, the correct

229 translation 'SCRATCHY' is paired with the foil translation 'CRAMPING'. It would not be

230 paired with a synonymous foil, like 'ITCHY', nor with an antonymic foil, like 'SMOOTH'.

231 Since not all of the meanings in the dataset have clear antonyms (a meaning like CRAMPING is

232 one example), this made the difficulty of the task comparable between different trials. Finally, 
Two iconicity measures are better than one

233 the length of the sentences was also kept consistent (to a difference of no more than 5 characters)

234 between pairings of translations and foils.

235 To test the robustness of the guessing procedure to different choices of translations and

236 foil translations, the same words were tested multiple times with two to three different correct

237 and foil translations. We were concerned that participants could choose English translations

238 based on how they sound (particularly if they sounded similar to the Japanese target word),

239 rather than based on their meanings as we intended. To minimise any potential effects from this,

240 as much as possible phonologically distinct synonyms were used as alternative translations

241 (e.g. SMALL|TINY, BIG|LARGE).

242 Pilot results from these guessing experiments showed that the guessability of the words

243 varied considerably depending on the particular combination of translations and foils used (see

244 Section 3.1). We hypothesised that, as well as considering the meaning of the English

245 translations and foils, participants may have been driven to one choose option over another

246 because of its phonetic similarity to the target word; because of lexical features of the word like

247 its frequency, valence, or arousal; or because of other associations they may have had with the

248 word. To address this, we developed a new guessing paradigm (described in Section 2.2.2) that

249 did not require participants to compare different English translations.

\section{2.2.2 Guessing experiments: meaning to word design}

251 In our novel guessing paradigm, the forced-choice aspect of the task was between Japanese

252 words rather than English translations. Participants were given a meaning (in English), and two

253 potential Japanese words for that meaning, then asked to guess which word matched the

254 meaning. By making the forced choice between two Japanese words that the participants did not 
Two iconicity measures are better than one

255 have any experience with, rather than between two familiar English words, we hoped to reduce

256 interference from factors other than iconicity that could influence the participant's forced-choice.

257 The instructions for the task were as follows:

We are interested in how well people can guess words in foreign languages. In this experiment, you will be asked to match the English translation with the corresponding

Listen to the Japanese words below.

One of them means WOBBLING

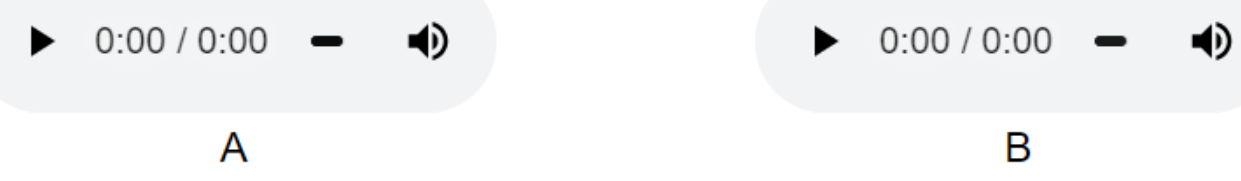

\section{Which one do you think means WOBBLING?}

Figure 2.2: Guessing task, meaning to word design.

263 This time, instead of using sentence frames for context, we kept the amount of English to a

264 minimum by using only single-word translations as much as possible. Where more context was

265 needed this was provided in brackets. A sample trial is shown in Figure 2.2.

266 The incorrect 'foil' word in each trial was a nonsense Japanese word artificially

267 generated to be as phonologically distinct as possible from the correct word, while still

268 conforming to Japanese phonotactics. For every word, three different foil words were generated 
Two iconicity measures are better than one

269 by substituting each consonant with its top three most phonologically distant consonants in the

270 Japanese sound system. Phonological distances were calculated using the feature matrix provided

271 in PHOIBLE (Moran \& McCloy, 2019), with the distance between two sounds being equal to the

272 sum of the distances between each of their feature values. Distances were calculated as follows:

The distance between two feature values that are identical is 0 . The distance between two opposing values (+/- or -/+) is 1-except if the feature involved is length or voicing, then the distance is 0.5 . The distance between two feature values when one of them is 0

277 Since /a/ is the most phonologically distant vowel from many of the vowels in the Japanese, to

278 avoid the foil words all having the same vowel, a different approach was used for substituting

279 vowels. Instead of using feature values, vowels were substituted with the vowel obtained by

280 rotating the vowels at the endpoints of the vowel space triangle 120 degrees clockwise or 281 counterclockwise, and flipping the vowels in the middle. So /u/ is substituted with either /a/ or $282 \mathrm{li} /$, /a/ is substituted with either /i/ or /u/, /i/ is substituted with either /a/ or /u/, and /e/ and /o/ are 283 substituted with each other (see Figure 2.3). Finally, reduplicated words were 'unreduplicated' 284 when creating foils. For example, the foils for fuwafuwa were watfi, nati and rid:u (not 285 watfiwat $i$, natinati and rid:urid:u). 

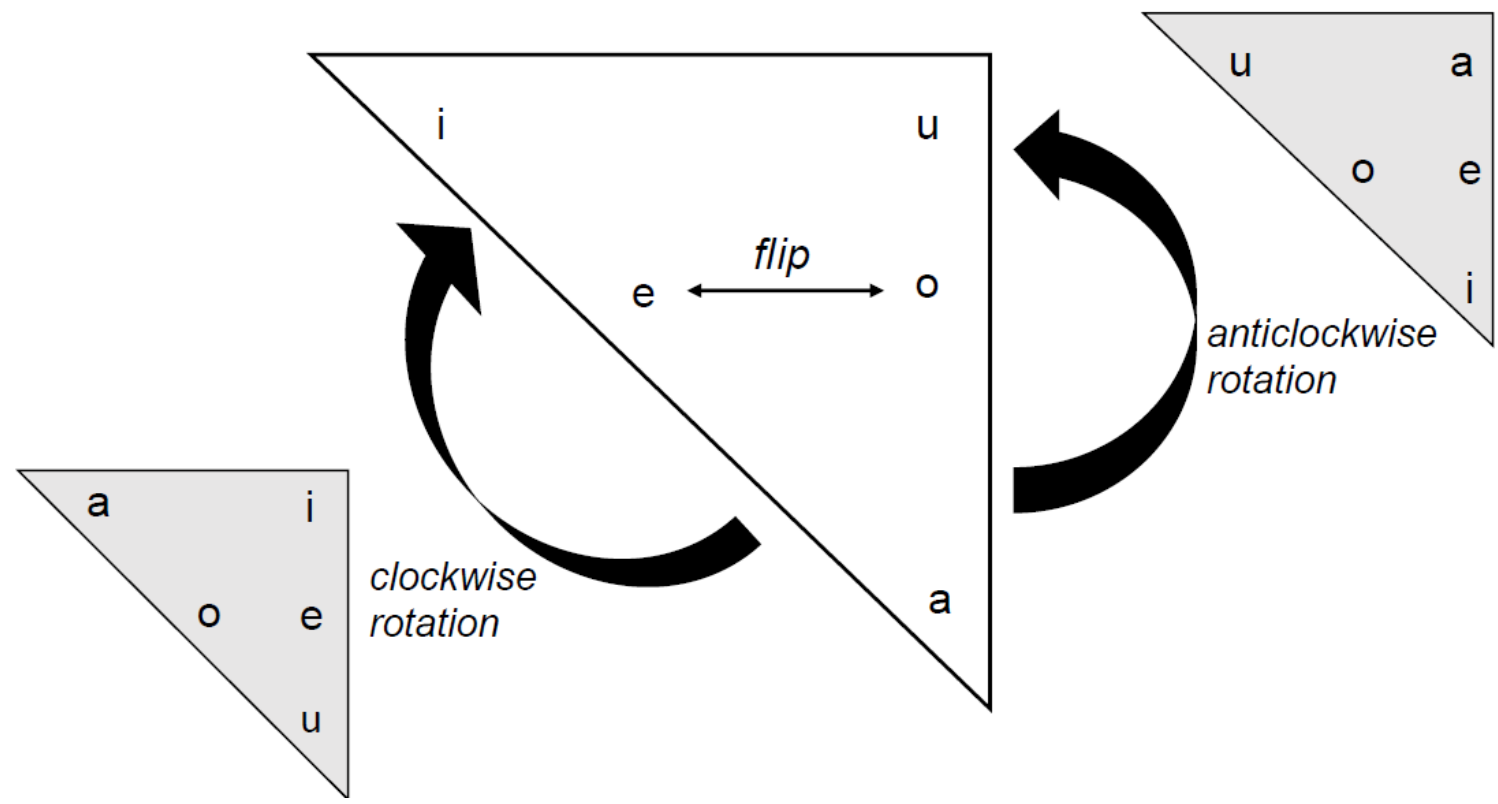

Figure 2.3: Vowel substitutions for the creation of foil words. Vowels were substituted with the vowel obtained by rotating the vowels at the endpoints of the vowel space triangle 120 degrees clockwise or counterclockwise, and flipping the vowels in the middle. So / $\mathrm{u} /$ is substituted with either $/ \mathrm{a} / \mathrm{or} / \mathrm{i} /$, /a/ is substituted with either /i/ or / $\mathrm{l} /$, /i/ is substituted with either /a/ or / $\mathrm{l} /$, and /e/ and /o/ are substituted with each other

By using foil words that sound as different as possible to the target word, we hoped to

293 improve the sensitivity of the guessing task to iconicity in the target word. Our logic was that if

294 the target word is iconic for a concept, then a word that sounds very different to the target word

295 should be a bad fit for that same concept-making the choice between the two easier. To test

296 whether this was really the case, we ran one of the experiments a second time using foils that

297 were randomly chosen from among the other words in the experiment, rather than phonologically

298 distinct foils. This allowed us to assess whether using phonologically distinct foils led to more

299 words being guessed correctly.

As with the previous guessing experiments, the same words were tested multiple times

301 with different translations and foils to assess the robustness of the results to the particular foil or

302 translation chosen. 
Two iconicity measures are better than one

303

304

305

306

307

308

309

321 Participants were instructed to listen to the Japanese words and were told their meanings. They

322 then had to rate the resemblance between the word and its meaning on a scale from 0 'No

323 resemblance' to 6 'Strong resemblance' (see Figure 2.4). Earlier studies have also included a

324 negative end of the scale, corresponding to a 'bad resemblance'. However, a meta-analysis found

325 that the negative end of the scale was both underused and inconsistently used, suggesting that 
Two iconicity measures are better than one

326 anti-iconic relationships are difficult to assess (Motamedi et al., 2019). For this reason, we used

327 only a positive scale in this study. As with the guessing studies, the translation shown for each

328 participant was varied randomly between a set of (wherever possible) phonologically distinct

329 synonyms.

Listen to the Japanese word below. It means WOBBLING.

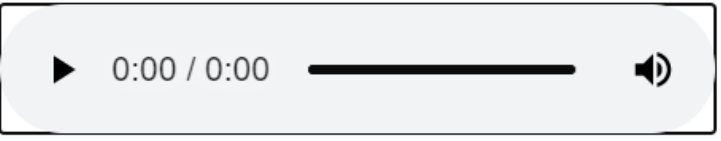

Do you think there is a resemblance between the word and its meaning?

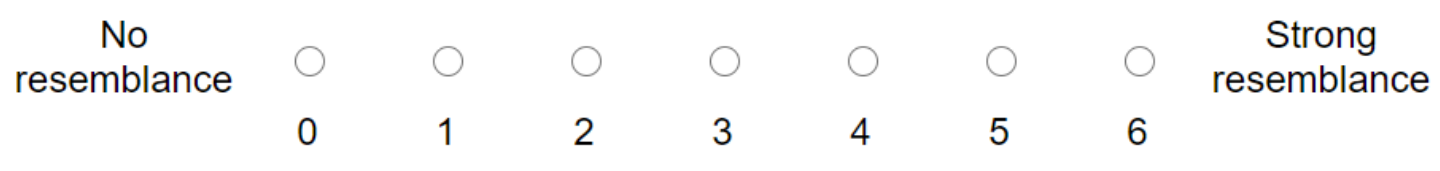

330

331

Figure 2.4: Rating task

\section{$332 \quad 3 \quad$ Results}

333 Statistical analyses were conducted using $\mathrm{R}$ version 4.2.1 (R Core Team 2022).

\section{$334 \quad 3.1$ Robustness to different choices of translations and foils}

335 We first investigated whether the results from the rating and guessing studies were robust to

336 different choices of translations (for both tasks) and foils (for the guessing tasks only). For each

337 word separately, we performed Fisher's Exact Tests on the guessing results, and ANOVAs on

338 the rating results, to determine whether there were significant differences in guessing accuracy or

339 ratings given depending on the translation or foil word shown. As the ANOVAs are less robust

340 to sparse data than the Fisher's Exact Tests, for the analysis of the rating data we excluded

341 translations with fewer than 10 ratings. 
Two iconicity measures are better than one

In the pilot version of the guessing task-where participants were guessing between

343 English translations-the guessability of 11 out of the 45 words tested $(=24 \%)$ differed

344 significantly depending on the translation and/or foil word chosen. By modifying the guessing

345 task so that participants guessed between Japanese words rather than English translations, we

346 were able to reduce this proportion to 37 out of 301 words tested $(=12 \%)$. This was comparable

347 to the variation found in the rating task when using different translations, which affected 11 out

348 of 88 words tested $(=13 \%)$. We looked into each of these cases individually, but could not find a

349 consistent explanation for the differing distributions of guesses and ratings. For the English

350 translations, frequency and arousal sometimes seemed to influence how often a particular

351 translation was chosen, or how highly the word-translation pairing was rated (in that more

352 frequent, high arousal English words seemed to be chosen more often and given higher iconicity

353 ratings). Resemblances between the sound of the translation words and the sound of the Japanese

354 words also sometimes seemed to play a role. However, we were unable to determine which of

355 these factors would be most relevant in any one case, nor how they would interact.

\section{$356 \quad 3.2$ Sensitivity and discriminability}

357 To test whether using phonologically distinct foils in the guessing experiments improved the

358 sensitivity of the task to iconicity, a subset of 30 words were tested twice- once with

359 phonologically distinct "opposite" foils, and once with random foils. The number of these words

360 which were guessed at rates significantly higher than chance (using an exact binomial test, with a

361 sample of 30 guesses per word an accuracy greater than or equal to $2 / 3$ is needed to be $95 \%$

362 confident the true accuracy is above chance) was greater when using opposite foils than when

363 using randomly selected foils. Using opposite foils, just over half of the words in the sample

364 (16/30) were guessed at rates significantly higher than chance, whereas with the random foils 
Two iconicity measures are better than one

365 this number fell to just 1 in 3 words (10/30). Only two words were guessed significantly higher

366 than chance in the random foil condition, but not in the opposite foil condition. However, both

367 were guessed numerically above chance in the opposite foil condition as well (with accuracies of

$36852 \%$ and 57\%). Figure 3.1 compares the distribution of guessing accuracies between the two

369 conditions. When using random foils, most of the distribution is centred around chance. When

370 using opposite foils, however, the guesses are more evenly dispersed across the full range of

371 accuracies. Critically, the portion of the distribution that is above chance is larger in the opposite

372 foil condition compared to the random foil condition.

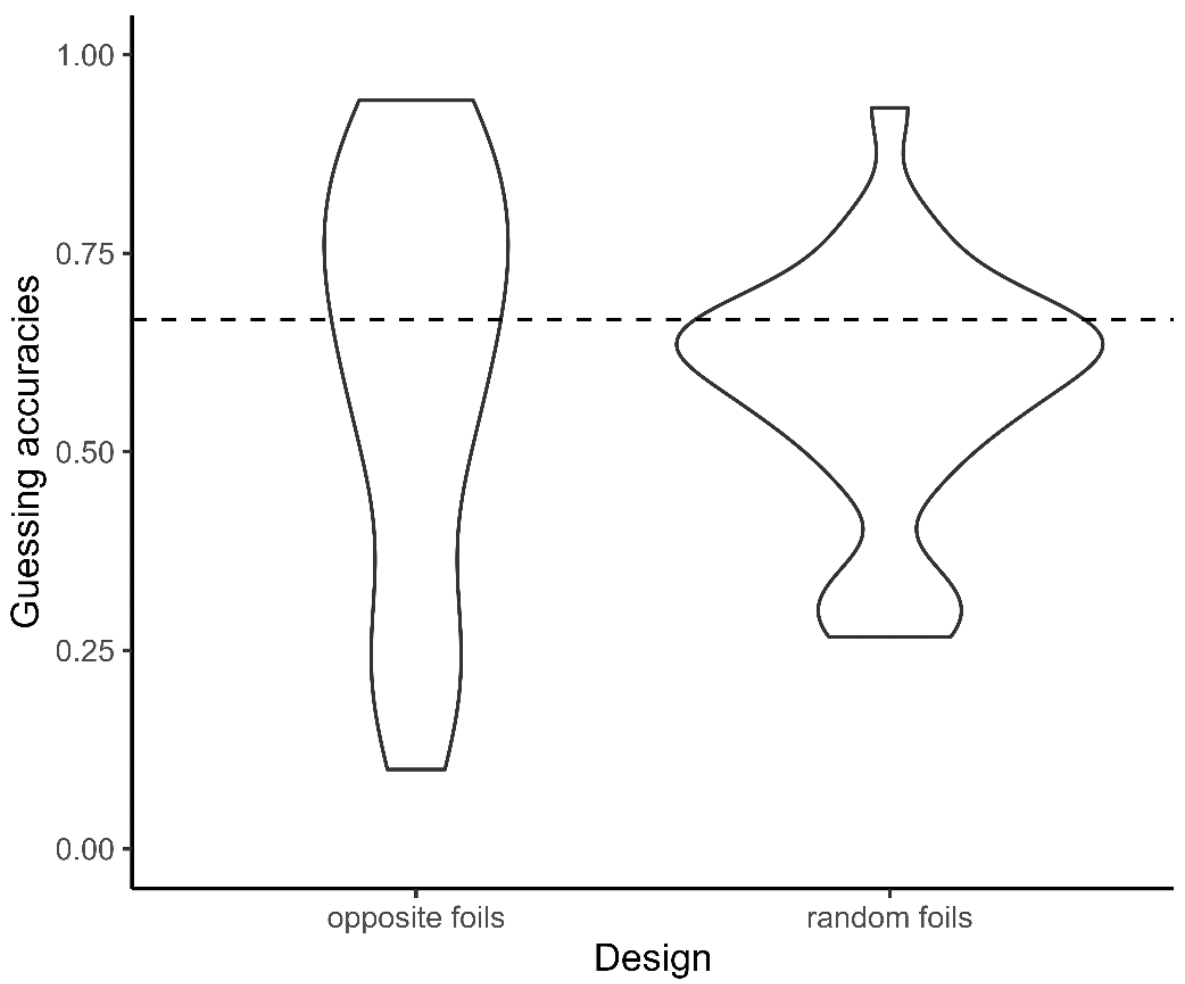

Figure 3.1: Guessing accuracies when using opposite versus random foils. The dotted line indicates the $\mathbf{9 5 \%}$ confidence interval for accuracies greater than chance.

We also compared the distribution of measures (guessing accuracies and iconicity

378 participants who did not speak any Japanese, whereas rating studies more commonly use native 
Two iconicity measures are better than one

379 speaker participants, we added one more plot to the figure showing the distribution of iconicity 380 ratings in a separate study by Thompson et al. (2020). Thompson et al. collected iconicity ratings

381 for Japanese words from native Japanese speaking participants. Their dataset contained a wider

382 variety of words than the current dataset, and they also used a different rating scale. Their scale

383 started at -5 for 'anti-iconic' words, and went up to 5 for 'iconic' words, with 0 indicating

384 arbitrariness. The scale in the current study started at 0 (also corresponding to an 'arbitrary'

385 category, in which there is "no resemblance between the word and its meaning"), and went up to

3866 to reflect a "strong resemblance between the word and meaning". To make the data more

387 comparable between the two studies, and since a meta-analysis found negative ratings unreliable

388 (Motamedi et al., 2019), we have filtered the data from Thompson et al. to only contain

389 responses using the part of the scale between 0 and 5. Also, since the Thompson et al. study

390 included loanwords, whereas the current study contained only native words, to improve

391 comparability between the studies we have also filtered the Thompson et al. data to remove non-

392 native words. Finally, ratings from both studies were transformed so that they varied between 0

393 and 1, allowing them to be compared with the guessing accuracies. The resulting distributions

394 are shown in Figure 3.2 
Two iconicity measures are better than one

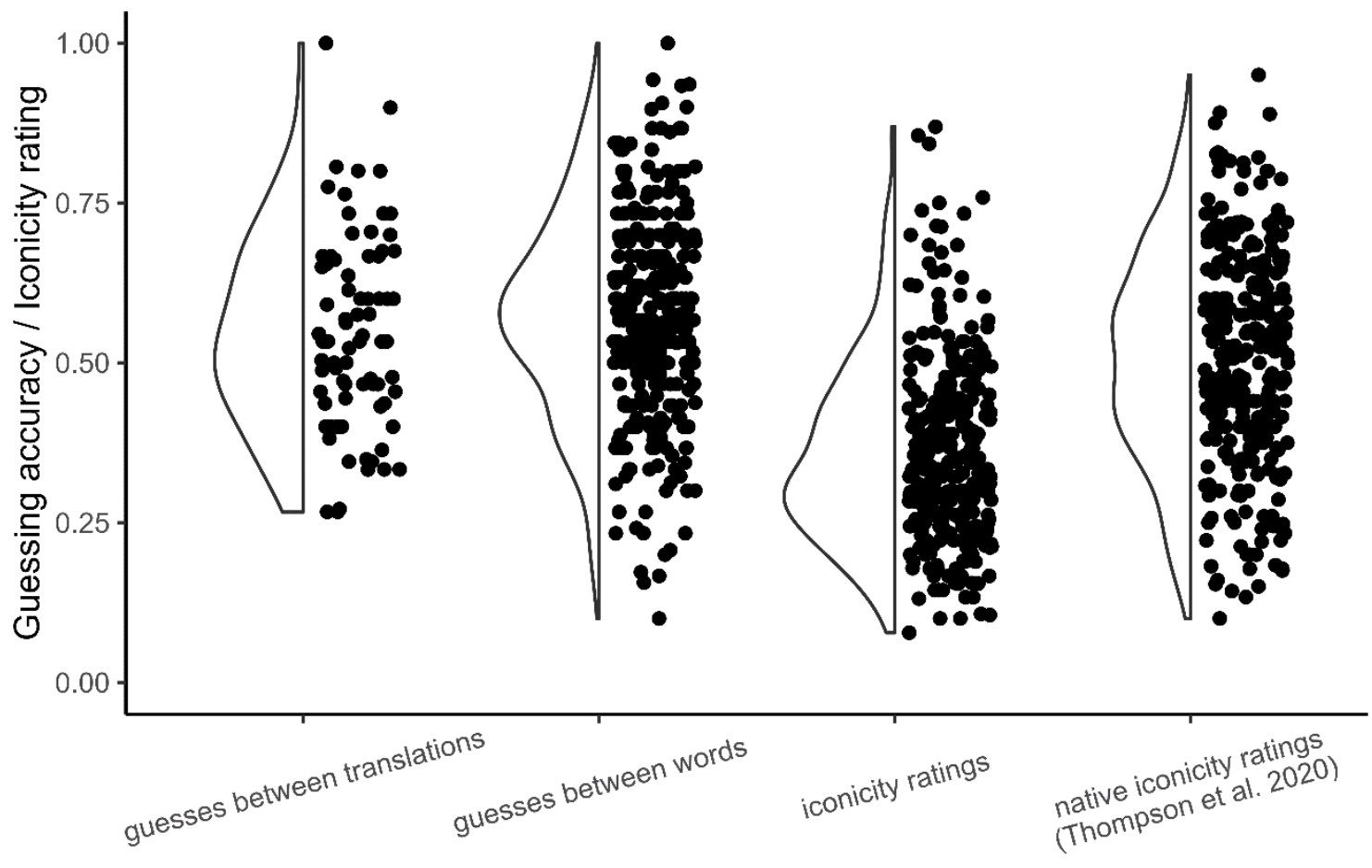

Method speakers, however, we can see that these are dispersed similarly to the guesses between words

404 from the current study. Their distribution is slightly narrower at the very top end of the scale.

405 However, the words in the current study were taken from a portion of the vocabulary (the 406 sensory lexicon) predicted to be high in iconicity (see e.g. Winter et al. 2017), whereas the data 407 from Thompson et al. was more varied, with proportionally fewer ideophones. So this could 
Two iconicity measures are better than one

408 simply be a reflection of underlying differences between the two datasets, rather than a reflection

409 of the sensitivity of the measures used.

\section{$410 \quad 3.3 \quad$ Agreement}

411 Figure 3.3 compares the guessing accuracies and mean iconicity ratings for ideophones and

412 prosaic words. For the guessing accuracies, the solid line represents chance while the dotted lines

413 above and below indicate the 95\% confidence interval for accuracies greater than or lesser than

414 chance respectively. The pilot guessing study where participants guessed between translations

415 contained different words to those used in the subsequent guessing and rating studies, so here

416 only the results from the later guessing study (where participants guessed between words) are

417 shown. At the top end of the scale, the two measures agree-ideophones score higher than prosaic

418 words for both ratings and guesses. However, while the bottom end of the scale is dominated by

419 prosaic words in the ratings, in the guessing results we again find ideophones at the extremities.

420 That is, ideophones were guessed both better and worse than prosaic words. Also, while both

421 ideophones and prosaic words were guessed at above-chance accuracies, the portion of the graph

422 corresponding to below-chance accuracies is dominated by ideophones. We will return to this in

423 the discussion. 

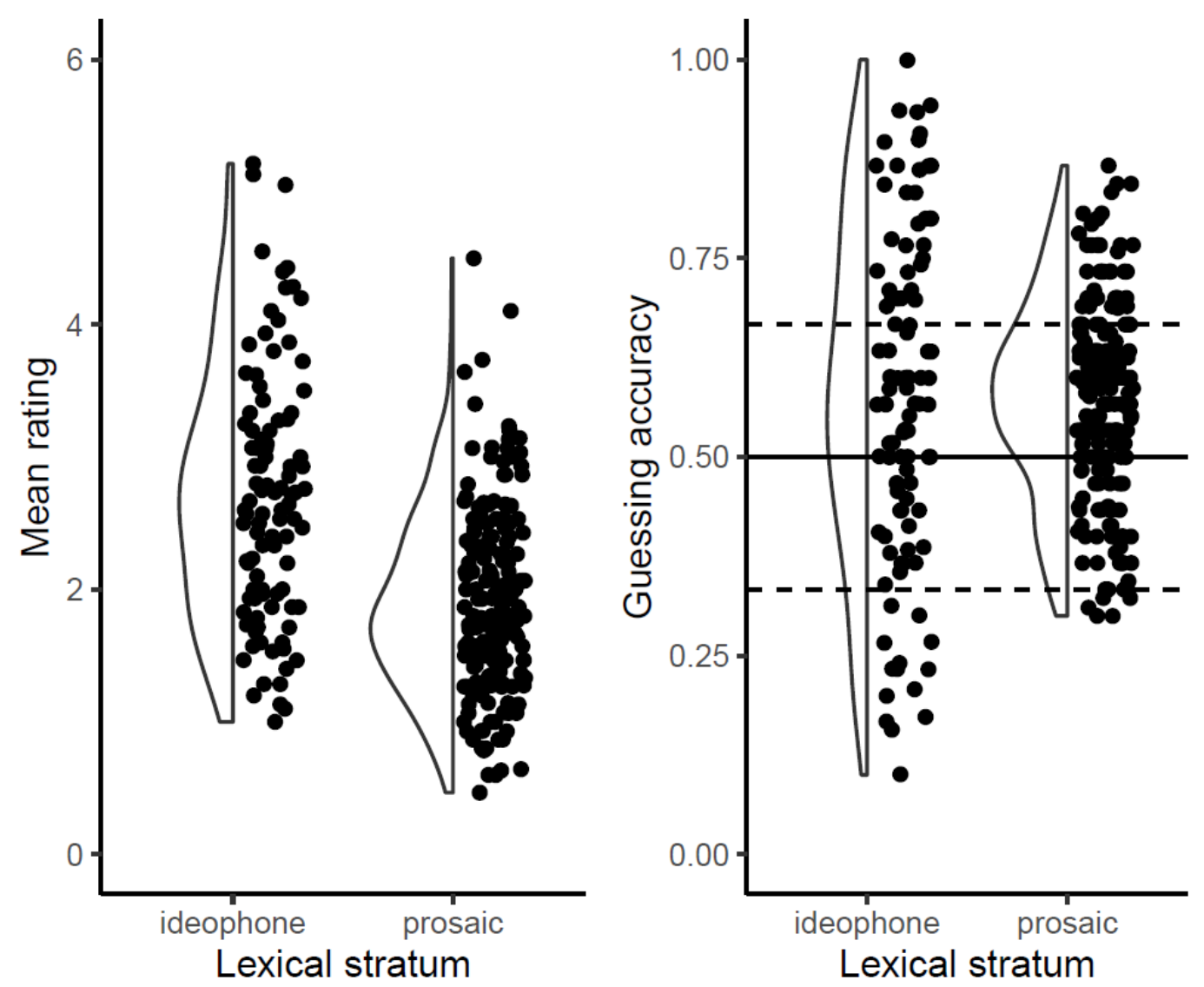

Figure 3.3: Comparison of iconicity ratings and guessing accuracies, for ideophones and prosaic words. For the guessing accuracies, the solid line indicates chance while the dotted lines above and below indicate the $\mathbf{9 5 \%}$ confidence interval for accuracies greater or lesser than chance, respectively.

To assess how well the measures agree on the iconicity of individual words, iconicity

431 ratings and guessability scores for the same words were transformed to z-scores so that they

432 could be directly compared. Figure 3.4 plots the z-scores for each measure against each other, to

433 show the agreement between them. In the figure, ideophones are represented by blue points, and

434 prosaic words by orange points. 
Two iconicity measures are better than one

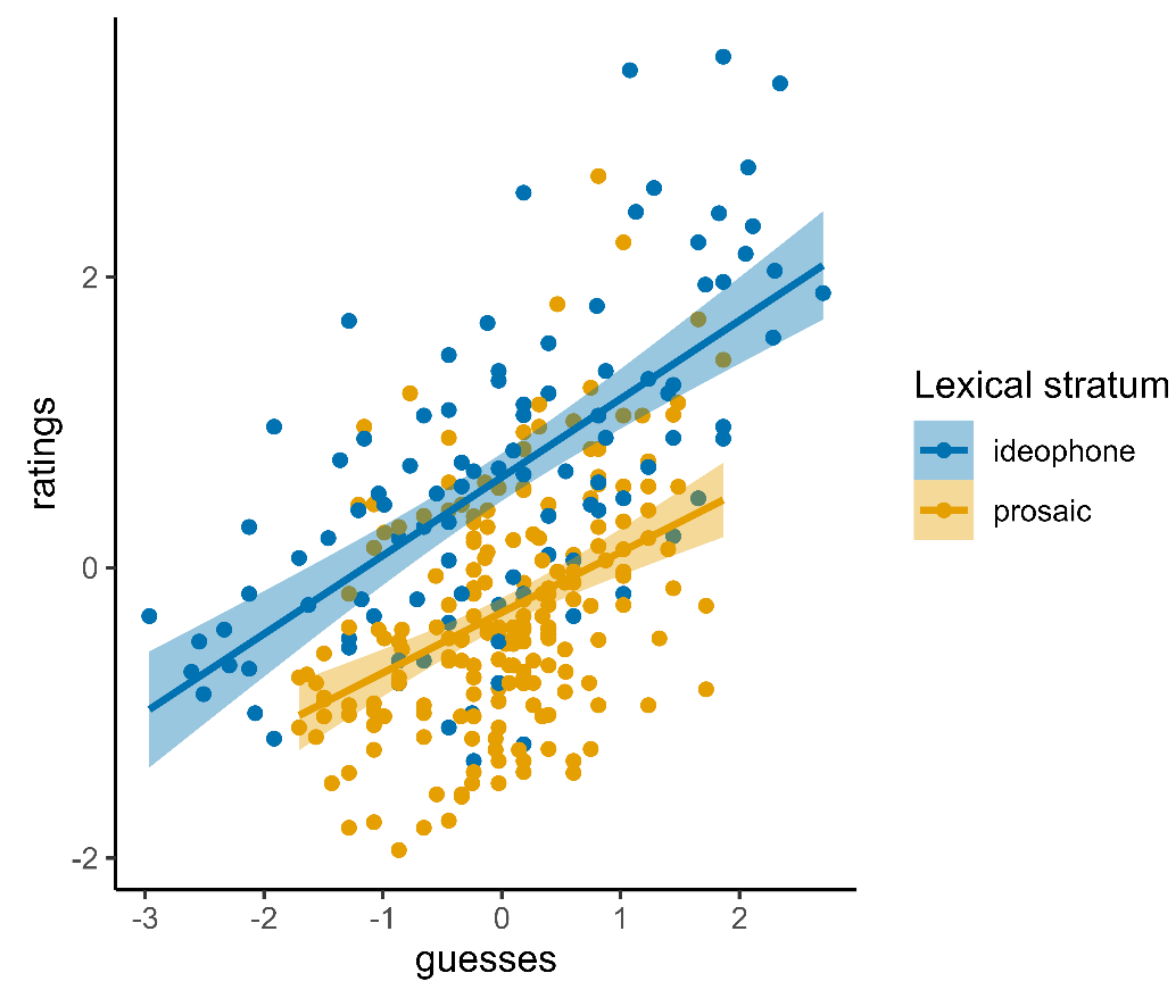

Figure 3.4: Agreement between guesses and ratings. Ideophones are represented by blue points, and prosaic words by orange points.

For both ideophones and prosaic words, iconicity ratings and guessing accuracies were

439 strongly correlated. This correspondence was slightly better for ideophones $(r=0.66,95 \% C I$

$440[0.44,0.79], 95 \%$ CI $[0.53,0.76], t(99)=8.68, p<.001)$ compared to prosaic words $(r=0.41$,

$44195 \%$ CI $[0.29,0.52], t(201)=6.45, p<.001)$. To explore this relationship further, we created

442 two linear regression models: one predicting ratings from guesses, lexical stratum, and the

443 interaction between these two factors; and a second model predicting guesses from ratings,

444 lexical stratum, and the interaction between these two factors. Again, both models used z-scores

445 rather than raw ratings and guesses. The models were created using the $\mathrm{R}$ package lmerTest

446 (version 3.1.3) (Kuznetsova et al., 2017). The output of the first model (predicting ratings from

447 guesses) is shown in Table 3.1. 
Two iconicity measures are better than one

\begin{tabular}{lcccc}
\hline \hline & \multicolumn{5}{c}{ Ratings } \\
\hline Predictors & Estimates & std. Error & Statistic & $p$ \\
\hline Intercept) & 0.62 & 0.08 & 8.26 & $<0.001$ \\
guesses & 0.54 & 0.06 & 9.52 & $<0.001$ \\
stratum [prosaic] & -0.93 & 0.09 & -10.10 & $<0.001$ \\
guesses * stratum & -0.12 & 0.09 & -1.40 & 0.163 \\
[prosaic] & & & \\
\hline Observations & \multicolumn{3}{c}{304} \\
$\mathrm{R}^{2} / \mathrm{R}^{2}$ adjusted & \multicolumn{2}{c}{$0.432 / 0.426$} &
\end{tabular}

451 The model shows that guessing accuracies predict iconicity ratings, in that the better

452 guessed a word was, the higher its iconicity rating. There was also an effect of lexical stratum,

453 such that prosaic words were predicted to receive lower iconicity ratings than ideophones with

454 the same guessing accuracies. There was no significant interaction between these two factors.

455 This is shown in Figure 3.5. The lines for ideophones and prosaic words are parallel, indicating

456 that differences in guesses correspond to differences in ratings in the same way for both

457 ideophones and prosaic words. However, the line for the ideophones is directly above the line for

458 prosaic words, indicating that ideophones are rated higher in iconicity than prosaic words-even

459 when guessed at the same accuracies. 


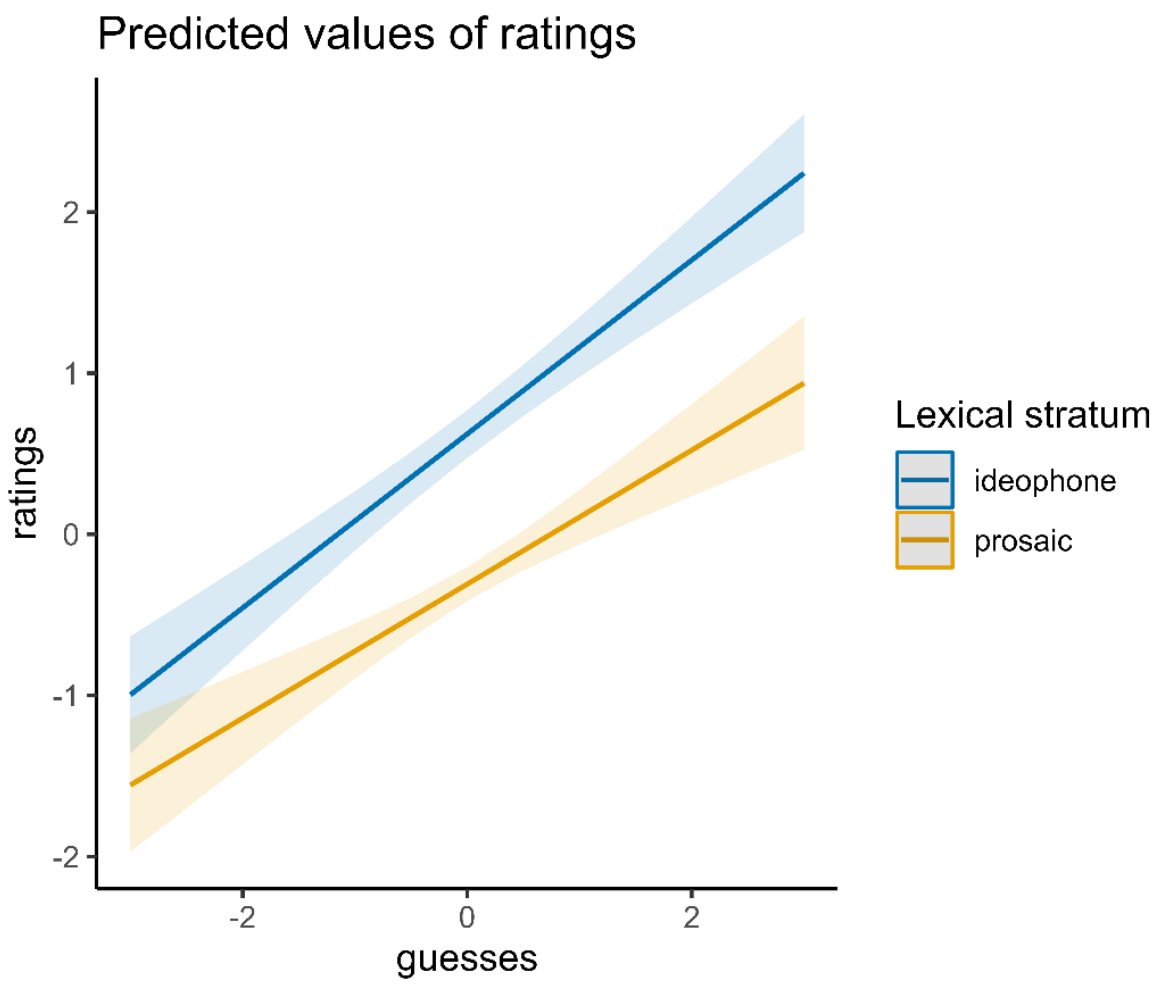

Figure 3.5: Relationship between lexical stratum, guessability, and predicted rating. The line for ideophones is shown in blue, and for prosaic words in orange.

Table 3.2 shows the output of the second model, in which we predicted guessing

464 accuracies from iconicity ratings. The model confirmed that the relationship between guesses

465 and ratings works both ways, with higher iconicity ratings also predicting higher guessing

466 accuracies. However, the relationship between ratings, guesses, and lexical stratum was more

467 complex than in the first model, as here we found an interaction effect in addition to the main

468 effects. These effects are easiest to interpret by plotting the interaction, shown in Figure 3.6.

Table 3.2: Linear regression model predicting guesses from ratings, lexical stratum, and the interaction between these two factors.

\begin{tabular}{lcccc}
\hline \hline & \multicolumn{4}{c}{ Guesses } \\
\hline Predictors & Estimates & std. Error & Statistic & $p$ \\
\hline (Intercept) & -0.51 & 0.09 & -5.36 & $<0.001$ \\
ratings & 0.80 & 0.08 & 10.65 & $<0.001$
\end{tabular}


Two iconicity measures are better than one

$\begin{array}{lllll}\text { stratum [prosaic] } & 0.64 & 0.11 & 5.65 & <0.001 \\ \begin{array}{l}\text { ratings * stratum } \\ \text { [prosaic] }\end{array} & -0.39 & 0.11 & -3.69 & <0.001\end{array}$

Observations

304

$R^{2} / R^{2}$ adjusted

$0.325 / 0.318$

471

472

473

474

475

476

477 ratings were also guessed well. For well-guessed items, there was no significant difference

478 between the ideophones and the prosaic words, as shown by the lines overlapping. However,

479 among the poorly guessed items, ideophones were guessed comparatively worse than prosaic

480 words with the same iconicity ratings. We will return to this in the discussion. 
Two iconicity measures are better than one

Finally, the lower correlation between guesses and ratings obtained for prosaic words

482 compared to ideophones suggests that participants may not have been as consistent or reliable

483 when rating prosaic words compared to when rating ideophones. To further investigate this, we

484 calculated person-total correlations (Curran, 2016) for the rating data using bootstrapping (5000

485 iterations; following Motamedi et al., 2019), for ideophones and prosaic words separately. Figure

4863.7 shows the agreement between raters (expressed as the correlation between each individual

487 rating, and the by-item average) for ideophones and prosaic words respectively. The dots

488 indicate the mean while the lines indicate $95 \%$ confidence intervals. Again, since these ratings

489 were from non-Japanese speakers, we added data from Japanese speakers from Thompson et

490 al. (2020) as a comparison.

491

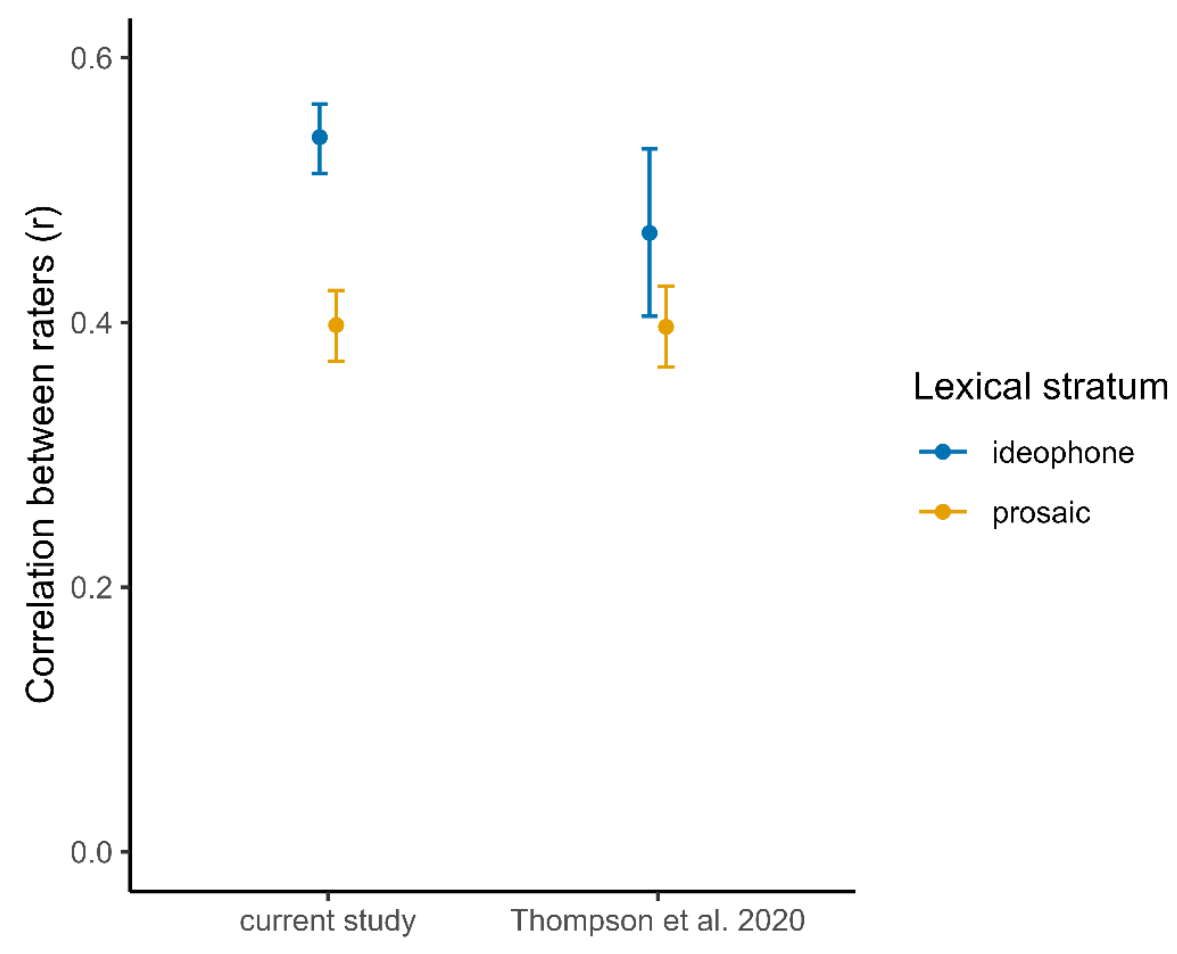

492

493 Figure 3.7: Consistency of iconicity ratings given for ideophones versus prosaic words, with mean and 494 $\mathbf{9 5 \%} \mathrm{Cls}$ indicated. Ideophones are shown in blue, and prosaic words in orange. 
Two iconicity measures are better than one

In both studies, participants were more consistent with each other when rating

496 ideophones than when rating prosaic words, though for Thompson et al. the overlap of the

497 confidence intervals indicates that the difference was not significant. There does not appear to be

498 any difference in the consistency of ratings between the English and the Japanese speakers, as

499 both the ideophone lines and the non-ideophone lines overlap between the two groups.

\section{Discussion}

\section{$501 \quad 4.1$ Relationship between ratings, guesses, ideophones, and iconicity}

502 The strong positive correlation between the ratings and the guesses, both for ideophones and for

503 prosaic words, confirms that these two measures tap into overlapping constructs, which we

504 suggest are form-meaning association biases. However, ideophones were consistently rated

505 higher in iconicity than prosaic words-even when guessed at the same accuracies (Figure 3.5).

506 We believe this is due to the majority of ideophones in the dataset being reduplicated. The two

507 lowest rated ideophones-sappari 'clean' and guttari 'exhausted'-were among the few in the data

508 that do not use a reduplicated template. Reduplication is common in onomatopoeic words in

509 English and is also a feature of child directed speech (Ferguson, 1978), in which iconicity plays a

510 prominent role (Perry et al., 2018). We suggest that because of these associations, the presence

511 of reduplication in the ideophones enhanced their perceived iconicity to English speaking

512 participants, above and beyond the influence of form-meaning associations themselves. This is in

513 line with previous work showing a relationship between iconicity ratings and structural

514 markedness (Dingemanse \& Thompson, 2020). The result is especially clear when we compare

515 the ratings with the guessing accuracies, particularly for poorly-rated ideophones, which were

516 guessed comparatively worse than prosaic words with the same ratings (Figure 3.6). While in the 
Two iconicity measures are better than one

517 rating task looking like an iconic word was enough to inflate ratings for ideophones (even in the

518 absence of form-meaning associations), this did not help participants to correctly guess their

519 meanings in the guessing task. For this, only form-meaning associations themselves were

520 helpful.

However, ideophones were still differentiated from prosaic words in the guessing results

522 in other ways. The most noticeable difference between the two word groups was that while both

523 were alternatively guessed at or above chance, of the words guessed at levels significantly below

524 chance almost all were ideophones (Figure 3.3). Perhaps because participants were more likely

525 to view form-meaning relationships in ideophones as meaningful (since they look like iconic

526 words), they attached more weight to perceived conflicts between a form and its given meaning

527 for this word group, resulting in a stronger pull away from the correct form (Dingemanse, 2019:

528 19; for more on this see discussion in Webster, 2017). This is supported by the larger person-

529 total correlations reported for ideophones compared to prosaic words in Section 3.3; that

530 participants were more consistent with each other when rating ideophones suggests that their

531 intuitions about form-meaning relations for these words were stronger.

532 So, although the structural markedness of the ideophones did not help participants to

533 decode their meanings, it did serve them in other ways. It led them to focus on the forms of the

534 ideophones and to view them as meaningful, which in turn meant their iconic intuitions about

535 these words were stronger (even if they did not always align with the association in Japanese).

536 As a first step in detecting iconic correspondences, this process (known as depiction marking;

537 see for discussion Clark (2016); Akita (2020); Dingemanse \& Akita (2017)) is frequently

538 overlooked, but here we see how important it can be, as without that step participants greatly

539 underestimated form-meaning associations in prosaic words. This is reflected in the finding that 
Two iconicity measures are better than one

540 prosaic words were guessed just as well as ideophones, even though they received lower

541 iconicity ratings. When forced to make a decision, participants must have used form-meaning

542 associations in the prosaic words to inform their guesses, but they did not recognise the presence

543 of these associations when rating the same words.

544 The result of some ideophones being very poorly guessed highlights another overlooked

545 aspect of iconicity, which is that construals of iconicity emerge from individual experience. How

546 is it that an ideophone which to a Japanese speaker so perfectly resembles its meaning, can to an

547 English speaker resemble the very opposite meaning? The finding highlights the role that

548 systematic patterns in the lexicon play in establishing form-meaning associations (Occhino et al.,

549 2017, 2020; Akita and Imai, 2022). English speakers without any knowledge of the Japanese

550 lexicon may not pick up on associations which to Japanese speakers feel very natural and

551 obvious, because unlike Japanese speakers they have seen these patterns thousands of times in

552 their own lexicon.

553 Alternatively, poor guessing accuracies and low iconicity ratings for some ideophones

554 could be explained by conflicting associations English speakers have with these forms, which

555 might not have anything to do with resemblance, but which can still influence perceptions of

556 form-meaning fit. As an example, one person commented that zarazara as a word for 'ROUGH'

557 sounds wrong to them because they associate the form zara with the clothing store ZARA, and

558 clothes are soft. Fortunately, the chances of English speakers having the same associations as

559 Japanese speakers with these forms through something not inherent to the forms themselves are

560 much smaller than the chance of them having a different association, so the highly-rated, well

561 guessed Japanese words likely do reflect form-meaning resemblances more than anything else. 
Two iconicity measures are better than one

562 However, the poorly guessed, poorly rated ideophones in particular highlight that experience also

563 plays a role in shaping construals of iconicity.

\section{$564 \quad 4.2$ Towards a synthesis of measures}

565 The above comparisons shed light on the variety of processes involved in meaning making

566 through iconicity, processes which do not take place in a vacuum but which interact with

567 different linguistic and cultural environments. To capture these processes, and tease them apart

568 in analyses, a synthesis of different measures focused on different aspects of iconicity offers

569 more than single shot approaches. For example, iconicity ratings from native speakers can tell us

570 which mappings are meaningful to them, while comparisons with guessability for non-speakers

571 can help to establish the relative contribution of (specific linguistic and cultural) experiences to

572 the meaningfulness of these mappings. This study used a single population of guessers, but

573 particularly for the poorly guessed items, a second or third group of guessers from a different

574 population would be helpful to ascertain whether specific items were poorly guessed because

575 their forms were not inherently meaningful, or whether conflicting associations among the

576 guessers led to poor guessing accuracies. In the latter case, a different group of guessers with

577 different experiences may not have the same trouble with these items.

578 Since ratings are more sensitive than guesses to the presentation of a word as iconic

579 through things like structural markedness, comparing the predictive strength of the two measures

580 could enable researchers to determine the degree to which processes like framing or staging are

581 involved in iconic effects, or whether the presence of form-meaning correspondences alone are

582 enough. Having guesses alongside ratings also makes the ratings themselves more interpretable.

583 For instance, the raters in this study rated all the words as "slightly" iconic, even though the 
Two iconicity measures are better than one

584 guessing results revealed many could not match the word to its meaning at rates any better than

585 chance. This is likely an artefact of the scale used in the rating task, where a lack of iconicity is

586 represented by just one of the seven points, with the other six all indicating varying degrees of

587 form-meaning resemblance. Correlating ratings with guessing accuracies offers a principled way

588 to determine what constitutes a 'low' or 'high' rating, as opposed to, e.g., arbitrarily dividing the

589 scale at the half-way point, or using percentiles tied to particular datasets.

\section{$590 \quad 4.3 \quad$ Methodological contributions}

591 Finally, we introduced and tested some modifications to the design of the guessing and rating

592 tasks to improve their robustness to different choices of translations and foils, and to fine-tune

593 the sensitivity of the guessing task to detecting form-meaning correspondences. We found that

594 participants were sometimes better at guessing the meaning of a word when a particular English

595 translation was chosen over another possible translation, or when it was paired with particular

596 foils. Iconicity ratings were similarly affected. For example, the ideophone pyonpyon was

597 guessed more accurately and rated higher in iconicity when translated as 'bouncing' rather than

598 'hopping'. These patterns appeared to be driven by multiple opaque factors that would be

599 difficult to control for, especially at large scales. To address this, we used multiple alternative

600 translations for the Japanese words in this study, showing different translations to different

601 participants. Where results are consistent across the different translations, we can assume the

602 effect is driven by the semantic content that the translations share, rather than by extraneous

603 features (e.g. frequency, arousal, etc.) that could differ between the translations. In the rating

604 task, we found roughly $90 \%$ of the ratings were robust to the choice of translation in this way.

605 However, using traditional guessing paradigms in which participants are presented with a word

606 and must choose its translation (a word-to-meaning design) this number fell to roughly $75 \%$. We 
Two iconicity measures are better than one

607 attributed this drop in robustness to the addition of more English translations in the guessing

608 task, which heightens the influence of extraneous features by introducing interactions between

609 the two alternative translations. To counter this, we developed a new guessing paradigm in which

610 participants are presented with only a single English translation, and asked to choose a Japanese

611 word to match it to-a meaning-to-word design more comparable to that of the rating task. We

612 found that this new paradigm increased the robustness of the guessing measures to roughly $90 \%-$

613 on par with the ratings.

614 As foil words for this new guessing paradigm, we could have used the other Japanese

615 words in the study, and randomly varied them between trials. However, because iconicity in

616 spoken language is fairly subtle, we developed a different approach which we hoped would

617 increase the sensitivity of the measure to detecting form-meaning resemblances specifically.

618 Rather than using real words as alternatives, we used artificial words constructed to be

619 maximally phonologically distinct from the Japanese words being tested. Our hypothesis was

620 that if the real word is a good fit for the meaning, then a word that sounds very different to it

621 should be a bad fit for the meaning. So using phonologically distinct foils should enhance the

622 task's sensitivity to iconicity. We confirmed this by retesting a subset of the words with

623 randomly chosen foils, and comparing the results with those obtained using phonologically

624 distinct foils. As expected, using phonologically distinct foils increased the number of items

625 guessed above chance.

626 We were also interested in the discriminability of the different measures. That is, how

627 well they distinguished between greater and lesser amounts of iconicity. We found that between

628 the two tasks, the guessing accuracies provided a wider spread of measures than the ratings. The

629 forced-choice nature of the guessing task meant participants had to act on even their weakest 
Two iconicity measures are better than one

630 intuitions, intuitions they may not have felt confident to express in the rating task. A lack of

631 confidence in rating foreign words is understandable, so we compared the ratings in this study

632 with ratings for Japanese words taken from native speakers in another study (Thompson et al.,

633 2020). We found that native speakers were more confident in giving high or low ratings, with the

634 distribution of native speaker ratings providing a comparable spread of measures to the guesses.

635 However, we did not find any difference in the consistency of the ratings provided between the

636 two groups-non-speakers were just as consistent as native speakers in rating the iconicity of

637 Japanese words. This supports the use of non-speaker ratings in situations where native speaker

638 ratings would be problematic (as in the current study; see discussion in Section 2.2), while

639 adding that ratings can be supplemented with guesses where more power is needed. Our new

640 guessing paradigm also helps in this case, as we found the meaning-to-word design increased the

641 range of guessing accuracies, with more words guessed at high (and low) accuracy levels, and

642 fewer words guessed at chance. This is probably because in the word-to-meaning design, in order

643 to make the difficulty comparable between trials, we had to restrict the foils to words from the

644 same sensory modality, which were neither synonyms nor antonyms of the correct translation

645 (Section 2.2.1). The meaning-to-word design eliminated this issue, making it easier for

646 participants to choose between the two different options, which resulted in a more discriminating

647 measure.

648 Having developed these methods for more robust, sensitive, and discriminating measures

649 of iconicity, we also packaged them in icotools (https://pypi.org/project/icotools/), so that

650 other researchers can benefit from them. Icotools can be used to automatically generate

651 guessing and rating tasks from standardised wordlists, following the designs in Section 2.2.2 and

652 Section 2.2.3. A full discussion of icotools and its functionality is provided in the 
Two iconicity measures are better than one

653 Supplementary Materials. As well as audio files, the package supports videos and images as

654 stimuli formats, making it suitable for investigations of iconicity in multiple modalities.

\section{Conclusions}

656 There has been a tendency in iconicity research to view resemblance as a silver bullet, but when

657 we really look into it resemblance alone does not always explain why, for example, smooch is

658 better than kiss, or smoosh is better than flatten. That resemblances exist is not always enough-

659 we also have to access them, and the things that determine whether and how we do this are likely

660 to be part and parcel of many iconic effects. This means that measures of iconicity also need to

661 be sensitive to the involvement of these other processes. Here we show how triangulating

662 insights from multiple measures offers a way to do this, as the comparison of results from rating

663 and guessing tasks reveals the influence of framing and experience on the different measures. As

664 the empirical study of ideophones and iconicity is coming of age, it is critical to couple

665 descriptive and theoretical work with quantification and reproducible methods. We made strides

666 towards this by exploring how to improve the reliability of rating and guessing measures, while

667 also making the guessing measures more targeted, and by packaging these improvements in

668 icotools, which simplifies and standardises the process of generating rating and guessing tasks.

669 We hope that the methods and findings presented here provide a foundation for future work to

670 build on, as well as a pointer towards a more nuanced treatment of iconicity as situated in

671 broader processes.

\section{Data Availability Statement}

673 The data from the study, as well as the code used to produce the experiments and analyses can be

674 found on the Open Science Framework at https://osf.io/j57uc/. 
Two iconicity measures are better than one

\section{Competing Interests Declaration}

676 The authors declare that the research was conducted in the absence of any commercial or

677 financial relationships that could be construed as a potential conflict of interest.

\section{Acknowledgments}

679 The authors would like to thank Ian Joo and Bodo Winter for providing the opportunity to

680 present an early version of the manuscript for feedback, as well as the three anonymous

681 reviewers for their thoughtful comments and advice.

\section{References}

683 Akita, K. (2008). Phonosemantic evidence for the mimetic stratum in the Japanese lexicon. Annual $684 \quad$ Meeting of the Berkeley Linguistics Society, 34, 1-12.

685 Akita, K. (2020). A typology of depiction marking: The prosody of Japanese ideophones and

686 beyond. https://doi.org/10.1075/sl.17029.aki

687 Akita, K. (2017). Grammatical and functional properties of mimetics in Japanese. In N. Iwasaki, P.

688 Sells, \& K. Akita (Eds.), The Grammar of Japanese Mimetics: Perspectives from structure,

689 acquisition and translation (pp. 20-34). Routledge.

690 Akita, K., \& Imai, M. (2022). The iconicity ring model for sound symbolism. Iconicity in Cognition $691 \quad$ and Across Semiotic Systems, 18, 27.

692 Blasi, D. E., Wichmann, S., Hammarström, H., Stadler, P. F., \& Christiansen, M. H. (2016). Sound693 meaning association biases evidenced across thousands of languages. Proceedings of the

$694 \quad$ National Academy of Sciences, 113(39), 10818-10823.

695 Boersma, P., \& Weenink, D. (2020). Praat: Doing phonetics by computer [Computer program]. 696 http://www.praat.org/

697 Brown, R. W., Black, A. H., \& Horowitz, A. E. (1955). Phonetic symbolism in natural languages. $698 \quad$ The Journal of Abnormal and Social Psychology, 50(3), 388.

699 Caselli, N. K., Sehyr, Z. S., Cohen-Goldberg, A. M., \& Emmorey, K. (2017). ASL-LEX: A lexical 700 database of American Sign Language. Behavior Research Methods, 49(2), 784-801. 701 Clark, H. H. (2016). Depicting as a method of communication. Psychological Review, 123(3), 324. 702 Curran, P. G. (2016). Methods for the detection of carelessly invalid responses in survey data.

$703 \quad$ Journal of Experimental Social Psychology, 66, 4-19.

704 Dingemanse, M. (2019). 'Ideophone' as a comparative concept. In K. Akita \& P. Pardeshi (Eds.), 705 Ideophones, mimetics, and expressives (pp. 13-33). John Benjamins Publishing Company. 706 Dingemanse, M., \& Akita, K. (2017). An inverse relation between expressiveness and grammatical 707 integration: On the morphosyntactic typology of ideophones, with special reference to 708 Japanese 1. Journal of Linguistics, 53(3), 501-532. 
Two iconicity measures are better than one

709 Dingemanse, M., Blasi, D. E., Lupyan, G., Christiansen, M. H., \& Monaghan, P. (2015).

710 Arbitrariness, iconicity, and systematicity in language. Trends in Cognitive Sciences, 19(10), 711 603-615.

712 Dingemanse, M., Schuerman, W., Reinisch, E., Tufvesson, S., \& Mitterer, H. (2016). What sound

713 symbolism can and cannot do: Testing the iconicity of ideophones from five languages.

$714 \quad$ Language, 92(2), e117-e133.

715 Dingemanse, M., \& Thompson, B. (2020). Playful iconicity: Structural markedness underlies the

716 relation between funniness and iconicity. Language and Cognition, 12(1), 203-224.

717 https://doi.org/10.1017/langcog.2019.49

718 Ferguson, C. A. (1978). Talking to children: A search for universals. Universals of Human

$719 \quad$ Language, 1, 203-224.

720 Fernández-Prieto, I., Navarra, J., \& Pons, F. (2015). How big is this sound? Crossmodal association

721 between pitch and size in infants. Infant Behavior and Development, 38, 77-81.

722 https://doi.org/10.1016/j.infbeh.2014.12.008

723 Fordyce, J. F. (1988). Studies in sound symbolism with special reference to English [PhD thesis].

$724 \quad$ University of California.

725 Fort, M., Lammertink, I., Peperkamp, S., Guevara-Rukoz, A., Fikkert, P., \& Tsuji, S. (2018).

726 Symbouki: A meta-analysis on the emergence of sound symbolism in early language

727 acquisition. Developmental Science, 21(5), e12659.

728 Hirayama, T. (1992). Gendai Nihongo Hōgen Daijiten [Dictionary of Contemporary Japanese

729 Dialects]. Meiji-shoin.

730 Hodge, G., \& Ferrara, L. (2022). Iconicity as Multimodal, Polysemiotic, and Plurifunctional.

$731 \quad$ Frontiers in Psychology, 13.

732 Iwasaki, N., David, P. V., \& Gabriella, V. (2007). What do English Speakers Know about gera-gera

733 and yota-yota?: A Cross-linguistic Investigation of Mimetic Words of Laughing and

$734 \quad$ Walking. Japanese Language Education Around the Globe; Japanese Language Education

$735 \quad$ Around the Globe, 17, 53-78.

736 Johansson, N. E., Anikin, A., Carling, G., \& Holmer, A. (2020). The typology of sound symbolism:

737 Defining macro-concepts via their semantic and phonetic features. Linguistic Typology,

738 24(2), 253-310.

739 Joo, I. (2020). Phonosemantic biases found in Leipzig-Jakarta lists of 66 languages. Linguistic

$740 \quad$ Typology, 24(1), 1-12.

741 Kageyama, T., \& Kishimoto, H. (Eds.). (2016). Handbook of Japanese Lexicon and Word

$742 \quad$ Formation. De Gruyter Mouton.

743 Köhler, W. (1929). Gestalt Psychology. Liveright.

744 Kunihira, S. (1971). Effects of the expressive voice on phonetic symbolism. Journal of Verbal

745 Learning and Verbal Behavior, 10(4), 427-429. https://doi.org/10.1016/S0022-

$746 \quad 5371(71) 80042-7$

747 Kuznetsova, A., Brockhoff, P. B., \& Christensen, R. H. B. (2017). lmerTest package: Tests in linear 748 mixed effects models. Journal of Statistical Software, 82(13), 1-26.

$749 \quad$ https://doi.org/10.18637/jss.v082.i13

750 Kwon, N. (2017). Empirically observed iconicity levels of English phonaesthemes. Public Journal 751 of Semiotics, 7(2), 73-93.

752 Little, H., Sulik, J., Cuskley, C., Flaherty, M., McCrohon, L., Ravignani, A., \& Verhoef, T. (2018).

$753 \quad$ What do iconicity judgements really mean. Proceedings of the 12th International

$754 \quad$ Conference on the Evolution of Language (Evolang12), 246, 248. 
Two iconicity measures are better than one

755 Magid, R. W., \& Pyers, J. E. (2017). "I use it when I see it": The role of development and 756 experience in Deaf and hearing children's understanding of iconic gesture. Cognition, 162, $757 \quad 73-86$.

758 McLean, B. (2022). JaponicSensoryLex. https://doi.org/10.5281/zenodo.5910236

759 Monaghan, P., Shillcock, R. C., Christiansen, M. H., \& Kirby, S. (2014). How arbitrary is language?

$760 \quad$ Philosophical Transactions of the Royal Society B: Biological Sciences, 369(1651), 76120130299.

762 Moran, S., \& McCloy, D. (Eds.). (2019). PHOIBLE 2.0. Max Planck Institute for the Science of $763 \quad$ Human History. https://phoible.org/

764 Motamedi, Y., Little, H., Nielsen, A., \& Sulik, J. (2019). The iconicity toolbox: Empirical

765 approaches to measuring iconicity. Language and Cognition, 11(2), 188-207.

766 Namy, L. L. (2008). Recognition of iconicity doesn't come for free. Developmental Science, 11(6),

767 841-846.

768 Occhino, C., Anible, B., \& Morford, J. P. (2020). The role of iconicity, construal, and proficiency in 769 the online processing of handshape. Language and Cognition, 12(1), 114-137.

770 Occhino, C., Anible, B., Wilkinson, E., \& Morford, J. P. (2017). Iconicity is in the eye of the

771 beholder. Gesture, 16(1), 99-125. https://benjamins.com/catalog/gest.16.1.04occ

772Peer, E., Rothschild, D., Gordon, A., Evernden, Z., \& Damer, E. (2022). Data quality of platforms

773 and panels for online behavioral research. Behavior Research Methods, 54(4), 1643-1662.

774 https://doi.org/10.3758/s13428-021-01694-3

775 Perlman, M., Little, H., Thompson, B., \& Thompson, R. L. (2018). Iconicity in Signed and Spoken

776 Vocabulary: A Comparison Between American Sign Language, British Sign Language,

$777 \quad$ English, and Spanish. Frontiers in Psychology, 9, 1433.

778 Perniss, P., Thompson, R., \& Vigliocco, G. (2010). Iconicity as a general property of language:

$779 \quad$ Evidence from spoken and signed languages. Frontiers in Psychology, 1(227), 1-15.

780 Perniss, P., \& Vigliocco, G. (2014). The bridge of iconicity: From a world of experience to the 781 experience of language. Philosophical Transactions of the Royal Society B: Biological $782 \quad$ Sciences, 369(1651), 20130300.

783 Perry, L. K., Perlman, M., \& Lupyan, G. (2015). Iconicity in English and Spanish and its relation to $784 \quad$ lexical category and age of acquisition. PloS One, 10(9), e0137147.

785 Perry, L. K., Perlman, M., Winter, B., Massaro, D. W., \& Lupyan, G. (2018). Iconicity in the speech $786 \quad$ of children and adults. Developmental Science, 21(3), e12572.

$787 \mathrm{R}$ Core Team. (2022). R: A language and environment for statistical computing. R Foundation for

$788 \quad$ Statistical Computing. https://www.R-project.org

789 Ramachandran, V. S., \& Hubbard, E. M. (2001). Synaesthesia-a window into perception, thought

$790 \quad$ and language. Journal of Consciousness Studies, 8(12), 3-34.

791 Sanada, S., \& Tomosada, K. (Eds.). (2015). Kenbetsu Hoogen Kanzyoo Hyoogen Jiten [Dialect

792 dictionary of emotion expressions by prefecture]. Tokyodo.

793 Sanada, S., \& Tomosada, K. (Eds.). (2018). Kenbetsu hoogen kankaku hyoogen jiten [Dialect

794 dictionary of sensory expressions by prefecture]. Tokyodo.

795 Sapir, E. (1929). A study in phonetic symbolism. Journal of Experimental Psychology, 12(3), 225.

796 Sevcikova Sehyr, Z., \& Emmorey, K. (2019). The perceived mapping between form and meaning in

$797 \quad$ American Sign Language depends on linguistic knowledge and task: Evidence from

798 iconicity and transparency judgments. Language and Cognition, 11(2), 208-234.

799 Shinohara, K., \& Kawahara, S. (2010). A cross-linguistic study of sound symbolism: The images of $800 \quad$ size. $36,396-410$. 
801 Taylor, I. K., \& Taylor, M. M. (1962). Phonetic symbolism in four unrelated languages. Canadian 802 Journal of Psychology/Revue Canadienne de Psychologie, 16(4), 344-356.

803 https://doi.org/10.1037/h0083261

804 Thompson, A. L., Akita, K., \& Do, Y. (2020). Iconicity ratings across the Japanese lexicon: A

$805 \quad$ comparative study with English. Linguistics Vanguard, O(0).

806 https://doi.org/10.1515/lingvan-2019-0088

807 Tsuru, S., \& Fries, H. (1933). A problem in meaning. Journal of General Psychology, 8, 281-284.

808 Tzeng, C. Y., Nygaard, L. C., \& Namy, L. L. (2017). Developmental change in children's sensitivity 809 to sound symbolism. Journal of Experimental Child Psychology, 160, 107-118.

810 Vinson, D. P., Cormier, K., Denmark, T., Schembri, A., \& Vigliocco, G. (2008). The British Sign 811 Language (BSL) norms for age of acquisition, familiarity, and iconicity. Behavior Research $812 \quad$ Methods, 40(4), 1079-1087.

813 Webster, A. K. (2017). " So it's got three meanings dil dil:" Seductive ideophony and the sounds of 814 Navajo poetry. The Canadian Journal of Linguistics/La Revue Canadienne de Linguistique, 815 62(2), 173-195.

816 Wichmann, S., Holman, E. W., \& Brown, C. H. (2010). Sound Symbolism in Basic Vocabulary.

$817 \quad$ Entropy, 12(4), 844-858. https://doi.org/10.3390/e12040844

818 Winter, B., \& Perlman, M. (2021a). Size sound symbolism in the English lexicon. Glossa: A

$819 \quad$ Journal of General Linguistics, 6(1). https://doi.org/10.5334/gjgl.1646

820 Winter, B., \& Perlman, M. (2021b). Iconicity ratings really do measure iconicity, and they open a 821 new window onto the nature of language. Linguistics Vanguard, 7(1).

822 Winter, B., Perlman, M., Perry, L. K., \& Lupyan, G. (2017). Which words are most iconic?

$823 \quad$ Interaction Studies, 18(3), 443-464.

824 Winter, B., Sóskuthy, M., Perlman, M., \& Dingemanse, M. (2022). Trilled /r/ is associated with

825 roughness, linking sound and touch across spoken languages. Scientific Reports, 12(1), 1035.

826 https://doi.org/10.1038/s41598-021-04311-7

827 Woods, K. J. P., Siegel, M., Traer, J., \& McDermott, J. H. (2017). Headphone screening to facilitate 828 web-based auditory experiments. Attention, Perception \& Psychophysics, 79(7), 2064-2072.

829 https://doi.org/10.3758/s13414-017-1361-2

830 